\title{
A new era for epigenetic epidemiology
}

\author{
Chathura J Gunasekara1 \& Robert A Waterland*,1,2 \\ 1 USDA/ARS Children's Nutrition Research Center, Department of Pediatrics, Baylor College of Medicine, Houston, TX 77030, USA \\ ${ }^{2}$ Department of Molecular \& Human Genetics, Baylor College of Medicine, Houston, TX 77030, USA \\ *Author for correspondence: waterland@bcm.edu
}

First draft submitted: 24 September 2019; Accepted for publication: 7 October 2019; Published online: 15 November 2019

Keywords: CORSIV • disease • DNA methylation • DOHaD • epigenetics • Illumina Infinium MethylationEPIC • Infinium Methylation $27 \mathrm{~K} \bullet$ Infinium Methylation $450 \mathrm{~K} \bullet$ metastable epiallele

Methylation of $\mathrm{CpG}$ dinucleotides is a mitotically heritable and highly stable epigenetic mechanism fundamental to cellular differentiation. Establishment of cell type-specific DNA methylation occurs throughout embryonic, fetal and early postnatal development, and stably regulates gene expression potential throughout life [1]. The ability of DNA methylation to stably regulate gene expression makes epigenetic and genetic etiology of disease equally plausible. Accordingly, since the first published use of the term in 2004 [2], there has been growing interest in the field of epigenetic epidemiology, defined as the study of the associations between interindividual epigenetic variation and risk of disease [3]. Because of its long-term stability and ability to be assayed in minute quantities of DNA, nearly all epigenetic epidemiologic studies have focused on $\mathrm{CpG}$ methylation. Compared with genetic epidemiology, epigenetic epidemiology presents major challenges [3]. The inherent cell-type specificity of DNA methylation means that we cannot generally 'epigenotype' an individual using peripheral blood DNA, complicating large-scale population studies of most human diseases. Also, even when the 'tissue of interest' can be obtained (in a case-control design, for example) [4-6], the disease process itself can induce epigenetic changes, compromising the ability to draw causal inferences.

The vast majority of so called 'epigenome-wide association studies' (EWAS) to date have employed Illumina methylation arrays. The earliest version, the Infinium HumanMethylation27 BeadChip (27 K) array, interrogated 27,578 CpG sites across 14,495 promoter regions of genes implicated in cancer. The subsequent Illumina HumanMethylation 450 BeadChip ( $450 \mathrm{~K}$ ) array was designed to measure methylation at approximately $450,000 \mathrm{CpG}$ sites covering $99 \%$ of RefSeq genes [7], facilitating comprehensive assessment of associations between tissue-specific differential methylation and gene expression [8]. In 2016 Illumina introduced an expanded version of the $450 \mathrm{~K}$ array, the HumanMethylationEPIC BeadChip (EPIC) array, which measures methylation at approximately 850,000 CpG sites, including $90 \%$ of those on the $450 \mathrm{~K}$ platform. The additional probes improved coverage of CpG sites in regulatory elements, including $58 \%$ of FANTOM5 enhancers, $7 \%$ of distal and $27 \%$ of proximal ENCODE regulatory elements [9].

We recognize the myriad advantages of a standardized commercial platform, the data from which can easily be compared across different studies, populations, etc. For population-level epigenetic epidemiology, however, the designs of the $450 \mathrm{~K}$ and EPIC arrays are far from ideal. Many of the probes on the $450 \mathrm{~K}$ and EPIC arrays cover CpGs that are generally not methylated in normal somatic tissues [9,10]. On both platforms, most probes do not show appreciable interindividual variation [10-12]. Consistent with these observations (and particularly troubling for studies attempting to prospectively predict later outcomes) a recent study using the EPIC array [12] observed generally poor test-retest reproducibility in peripheral blood DNA of the same individuals over a 1-year period.

A logical approach to overcome these limitations is to focus on genomic regions that display systemic interindividual variation (SIV) in DNA methylation [13,14]. Gunasekara et al. recently reported the largest SIV screen to date [15]. Previous studies identifying SIV regions [13,14,16] pursued the specific goal of identifying metastable epialleles [17], that is, stochastic (nongenetically mediated) systemic epigenetic variants. Given the difficulty of ruling out genetic influences in outbred human populations, Gunasekara et al. focused on identifying regions of SIV regardless of whether they are genetically or stochastically mediated. A computational algorithm was developed to analyze deep whole-genome bisulfite-sequencing (WGBS) data on tissues representing all three germ layers (thy- 
roid, heart and brain) from each of ten donors from the NIH Genotype-Tissue Expression (GTEx) project. The authors identified 9926 correlated regions of systemic interindividual variation (CoRSIVs) in the human genome. CoRSIVs, although involving just $0.1 \%$ of the genome, are intercorrelated over long genomic distances, associated with transposable elements and sub-telomeric regions, conserved across diverse human ethnic groups, sensitive to periconceptional environment and associated with genes implicated in a wide range of human disorders and phenotypes [15]. Remarkably, only $1 \%$ of $450 \mathrm{~K}$ or EPIC probes assay CpG sites within CoRSIV regions, rendering these platforms nearly blind to SIV in the human genome. This is extremely unfortunate given their preponderance among EWAS reported in the last decade. A database of published EWAS data (EWASdb) [18] currently hosts about $900450 \mathrm{~K}$ and 65 EPIC datasets.

Analogous to genetic variants, CoRSIVs are stable, systemic interindividual variants - essentially epigenetic polymorphisms. Notably, in the last year or two, previously identified regions of SIV (candidate metastable epialleles) have gained the attention of epigenetic epidemiologists, who have uncovered links to disease based on approximately 150 previously identified candidate metastable epialleles $[13,14,16]$. For instance, DNA methylation at SIV regions has recently been associated with Alzheimer's disease [19], autism [20,21], cancer [22], cleft palate [23], obesity [24], Parkinson's disease [6], rheumatoid arthritis [25] and schizophrenia [26]. These associations were among top hits in many cases, despite the underrepresentation of SIV regions on the Illumina arrays. Although causality remains unclear in most cases, prospective studies have identified predictive associations providing evidence of causality. For example, at SIV regions, DNA methylation in peripheral blood of healthy adults is associated with cancer diagnosed years later [27], and methylation in newborn blood samples is associated with BMI at age 5 [28]. Individual DNA methylation at metastable epialleles is stable over time [12], over 10 years in one example [13], underscoring the potential for CoRSIV methylation to contribute to disease prediction, diagnosis and prognosis. Further, SIV regions are particularly sensitive to periconceptional environment [14-16,29,30], making them an obvious focus for studies of the developmental origins of health and disease [3,31].

In summary, unlike the vast majority of HM450 and EPIC probe CpGs, CoRSIVs are systemic, highly variable among individuals, and stable over time within individuals. Focusing on these regions, investigators can employ genomic DNA from easily obtainable tissues like peripheral blood to draw inferences about epigenetic regulation throughout the body - akin to genotyping sequence variants. Although CoRSIVs are grossly underrepresented on the $450 \mathrm{~K}$ and EPIC arrays, accumulating data from these established platforms indicate that individual epigenetic variation at CoRSIVs is associated with a wide range of human diseases. To launch a new era in understanding how interindividual epigenetic variation affects risk of disease, we encourage others in the worldwide epigenomics community to join us in advocating for development of a standardized commercial platform focused on human CoRSIVs.

Financial \& competing interests disclosure

R Waterland is supported by USDA/ARS (CRIS 3092-5-001-059), the Cancer Prevention and Research Institute of Texas (grant number RP170295), and the National Institutes of Health (grant number 1R01DK111522). Where authors are identified as personnel of the Baylor College of Medicine, Houston, TX, USA, the authors alone are responsible for the views expressed in this article and they do not necessarily represent the decisions, policy or views of the Baylor College of Medicine, Houston, TX, USA. The authors have no other relevant affiliations or financial involvement with any organization or entity with a financial interest in or financial conflict with the subject matter or materials discussed in the manuscript apart from those disclosed.

No writing assistance was utilized in the production of this manuscript.

\section{Open access}

This work is licensed under the Attribution-NonCommercial-NoDerivatives 4.0 Unported License. To view a copy of this license, visit http://creativecommons.org/licenses/by-nc-nd/4.0/

\section{References}

1. Dor Y, Cedar H. Principles of DNA methylation and their implications for biology and medicine. Lancet 392(10149), 777-786 (2018).

2. Jablonka E. Epigenetic epidemiology. Int. J. Epidemiol. 33(5), 929-935 (2004).

3. Waterland RA, Michels KB. Epigenetic epidemiology of the developmental origins hypothesis. Annu. Rev. Nutr. 27, 363-388 (2007).

4. Viana J, Hannon E, Dempster E et al. Schizophrenia-associated methylomic variation: molecular signatures of disease and polygenic risk burden across multiple brain regions. Hum. Mol. Genet. 26(1), 210-225 (2017). 
5. Wang L, Fu X, Peng X et al. DNA Methylation Profiling Reveals Correlation of Differential Methylation Patterns with Gene Expression in Human Epilepsy. J. Mol. Neurosci. 59(1), 68-77 (2016).

6. Young JI, Sivasankaran SK, Wang L et al. Genome-wide brain DNA methylation analysis suggests epigenetic reprogramming in Parkinson disease. Neurol. Genet. 5(4), e342 (2019).

7. Bibikova M, Barnes B, Tsan C et al. High density DNA methylation array with single CpG site resolution. Genomics 98(4), 288-295 (2011).

8. Lokk K, Modhukur V, Rajashekar B et al. DNA methylome profiling of human tissues identifies global and tissue-specific methylation patterns. Genome Biol. 15(4), r54 (2014).

9. Pidsley R, Zotenko E, Peters TJ et al. Critical evaluation of the Illumina MethylationEPIC BeadChip microarray for whole-genome DNA methylation profiling. Genome Biol. 17(1), 208 (2016).

10. Grundberg E, Meduri E, Sandling JK et al. Global analysis of DNA methylation variation in adipose tissue from twins reveals links to disease-associated variants in distal regulatory elements. Am. J. Hum. Genet. 93(5), 876-890 (2013).

11. Hannon E, Lunnon K, Schalkwyk L, Mill J. Interindividual methylomic variation across blood, cortex, and cerebellum: implications for epigenetic studies of neurological and neuropsychiatric phenotypes. Epigenetics 10(11), 1024-1032 (2015).

12. Zaimi I, Pei D, Koestler DC et al. Variation in DNA methylation of human blood over a 1-year period using the Illumina MethylationEPIC array. Epigenetics 13(10-11), 1056-1071 (2018).

13. Silver MJ, Kessler NJ, Hennig BJ et al. Independent genomewide screens identify the tumor suppressor VTRNA2-1 as a human epiallele responsive to periconceptional environment. Genome Biol. 16, 118 (2015).

14. Waterland RA, Kellermayer R, Laritsky E et al. Season of conception in rural gambia affects DNA methylation at putative human metastable epialleles. PLoS Genet. 6(12), e1001252 (2010).

15. Gunasekara CJ, Scott CA, Laritsky E et al. A genomic atlas of systemic interindividual epigenetic variation in humans. Genome Biol. 20(1), 105 (2019).

16. Kessler NJ, Waterland RA, Prentice AM, Silver MJ. Establishment of environmentally sensitive DNA methylation states in the very early human embryo. Sci. Adv. 4(7), eaat2624 (2018).

17. Rakyan VK, Blewitt ME, Druker R, Preis JI, Whitelaw E. Metastable epialleles in mammals. Trends Genet. 18(7), 348-351 (2002).

18. Liu D, Zhao L, Wang Z et al. EWASdb: epigenome-wide association study database. Nucleic Acids Res. 47(D1), D989-D993 (2019).

19. Sanchez-Mut JV, Heyn H, Silva BA et al. PM20D1 is a quantitative trait locus associated with Alzheimer's disease. Nat. Med. 24(5), 598-603 (2018).

20. Dall'aglio L, Muka T, Cecil CAM et al. The role of epigenetic modifications in neurodevelopmental disorders: A systematic review. Neurosci. Biobehav. Rev. 94, 17-30 (2018).

21. Zhu Y, Mordaunt CE, Yasui DH et al. Placental DNA methylation levels at CYP2E1 and IRS2 are associated with child outcome in a prospective autism study. Hum. Mol. Genet. 28(16), 2659-2674 (2019).

22. Yang Y, Wu L, Shu XO et al. Genetically predicted levels of DNA methylation biomarkers and breast cancer risk: data from 228,951 women of European descent. J. Natl Cancer Inst. doi:10.1093/jnci/djz109 (2019).

23. Gonseth S, Shaw GM, Roy R et al. Epigenomic profiling of newborns with isolated orofacial clefts reveals widespread DNA methylation changes and implicates metastable epiallele regions in disease risk. Epigenetics 14(2), 198-213 (2019).

24. Kuhnen P, Handke D, Waterland RA et al. Interindividual variation in DNA methylation at a putative POMC metastable epiallele is associated with obesity. Cell Metab. 24(3), 502-509 (2016).

25. Mok A, Rhead B, Holingue C et al. Hypomethylation of CYP2E1 and DUSP22 promoters associated with disease activity and erosive disease among rheumatoid arthritispatients. Arthritis Rheumatol. 70(4), 528-536 (2018).

26. Boks MP, Houtepen LC, Xu Z et al. Genetic vulnerability to DUSP22 promoter hypermethylation is involved in the relation between in utero famine exposure and schizophrenia. NPJ Schizophr. 4(1), 16 (2018).

27. Van Baak TE, Coarfa C, Dugue PA et al. Epigenetic supersimilarity of monozygotic twin pairs. Genome Biol. 19(1), 2 (2018).

28. Van Dijk SJ, Peters TJ, Buckley M et al. DNA methylation in blood from neonatal screening cards and the association with BMI and insulin sensitivity in early childhood. Int. J. Obes. (Lond.) 42(1), 28-35 (2018).

29. Dominguez-Salas P, Moore SE, Baker MS et al. Maternal nutrition at conception modulates DNA methylation of human metastable epialleles. Nat. Commun. 5, 3746 (2014).

30. Estill MS, Bolnick JM, Waterland RA, Bolnick AD, Diamond MP, Krawetz SA. Assisted reproductive technology alters deoxyribonucleic acid methylation profiles in bloodspots of newborn infants. Fertil. Steril. 106(3), 629-639 (2016).

31. Waterland RA, Garza C. Potential mechanisms of metabolic imprinting that lead to chronic disease. Am. J. Clin. Nutr. 69(2), 179-197 (1999). 